\title{
A Neuro-Fuzzy Scheme for Integrated Input Fuzzy Set Selection and Optimal Fuzzy Rule Generation for Classification
}

\author{
Santanu Sen ${ }^{1}$ and Tandra $\mathrm{Pal}^{2}$ \\ ${ }^{1}$ Tejas Networks India Ltd., Bangalore - 560078, India \\ santanusen_82@yahoo.co.in \\ ${ }^{2}$ Department of Computer Science and Engineering, \\ National Institute of Technology, Durgapur 713209, India \\ tandranit@yahoo.com
}

\begin{abstract}
This paper proposes a scheme for designing a classier along with fuzzy set selection. It detects discontinuities in the domain of input and prunes the corresponding fuzzy sets using a neuro-fuzzy architecture. This reduces the size of the network and so the number of rules. The network is trained in three phases. In the rst phase, the network learns the important fuzzy sets. In the subsequent phases, the network is pruned to produce optimal rule set by pruning conicting and less significant rules. We use a four-layered feed-forward network and error back propagation learning. The second layer of the network learns a modulator function for each input fuzzy set that identies the unnecessary fuzzy sets. In this paper, we also introduced the notion of utility factors for fuzzy rules. Rules with small utility factors are less signicant or less used rules and can be eliminated. They are learned and detected by the third layer. After training in phase 3 in its reduced structure, the system retains almost the same level of performance. The proposed system has been tested on synthetic data set and found to perform well.
\end{abstract}

\section{Introduction}

A classifier assigns a class label to an object taking feature vector of the object as input. Fuzzy systems implemented with neural networks are known as neural fuzzy systems [1]-4]. Fuzzy rule based systems have been successfully applied to various classication tasks [4, 6], 8]. The neuro-fuzzy systems proposed so far for function approximation [1]-3] or classication [4 are capable of learning a mapping from inputs to their corresponding outputs and/or feature selection. But they are not capable of analyzing the input data to nd discontinuities and utilizing this information to nd unnecessary fuzzy sets in reducing the size of the system. In [7, Krishnapuram and Lee uses fuzzy aggregation function to develop a neural network for classication. Feature selection is also done in their network in certain conditions.

Shann and $\mathrm{Fu}[2]$ proposed a fuzzy neural network (FNN) for selection of rules in a fuzzy controller. Initially, the network contained all possible fuzzy rules. The 
redundant rules were pruned from the system to get a concise rule base after EBP learning. Pal and Pal [1] discussed various limitations of the network proposed by Shann and $\mathrm{Fu}$ and provided a better scheme to maintain nonnegative certainty factors of rules for learning and rule pruning. Chakraborty and Pal $[3]$ proposed a scheme for simultaneous feature selection with system identication in neuro-fuzzy paradigm. In their network they used a special modulator function for each input feature to identify the redundant features. In another scheme for classication, they 4] used the same strategy for feature selection. Certainty factors are a measure of the condence in a rule, but they have used it as means of identifying a rule as a less used one. We train the network in three phases. In phase 1, the network is trained with all the nodes and links. After learning, the redundant fuzzy sets are discarded from the network. In phase 2, the system is retrained keeping only the impor- tant fuzzy sets. After training, the inconsistent (rules with the same antecedent but dierent consequences) and less signicant (rules with small utility factors) rules are pruned. The network is retrained to adjust its weights in its reduced architecture In phase 3. The paper is organized as follows. In section 2, we present the network structure of the proposed system. In section 3 , the learning scheme used by the network for fuzzy set selection and also for rule generation is discussed. Section 4 gives the simulation results. We conclude in section 5

\section{Network Structure}

\subsection{Network Structure for System Identification and Pattern Classification}

Let there be $s$ input features $\left(x_{1}, x_{2}, \ldots, x_{s}\right)$ and $c$ classes $\left(t_{1}, t_{2}, \ldots, t_{c}\right)$. For a given sample $x$ in the $s$ dimentional input feature space, the proposed network can deal with fuzzy rules of the form.

$R_{i}$ : If $x_{1}$ is $A_{1 i}$ and $x_{2}$ is $A_{2 i} \ldots$ and $x_{s}$ is $A_{s i}$ then $x$ belongs to class $t_{l}$ with a certainty $d_{l},(1 \leq l \leq c)$. Here, $A_{j i}$ is the $i$ th fuzzy set defined on the domain of $x_{j}$.

The neural fuzzy system is realized using a four-layered feed forward network which is quite similar to the network structure used in 4. The functions of the nodes with their inputs and outputs are discussed below layer by layer. We use suffixes $p, n, m$ and $l$ to denote the suffixes of the nodes corresponding to the layers 1 through 4 respectively. The output of each node is denoted by $z$. The total number of nodes in layer $i$ is denoted by $N^{i}$.

Each node in layer 1 represents an input linguistic variable and works as a buffer and transmits the input to the next layer. Hence, if $x_{p}$ is the input to a node of this layer, the output of the layer is

$$
z_{p}=x_{p}
$$

Primarily, the nodes in layer 2 act as fuzzifiers. Hence, if there are $N_{i}$ fuzzy sets associated with the $i$ th input feature and there are $s$ input features then the 


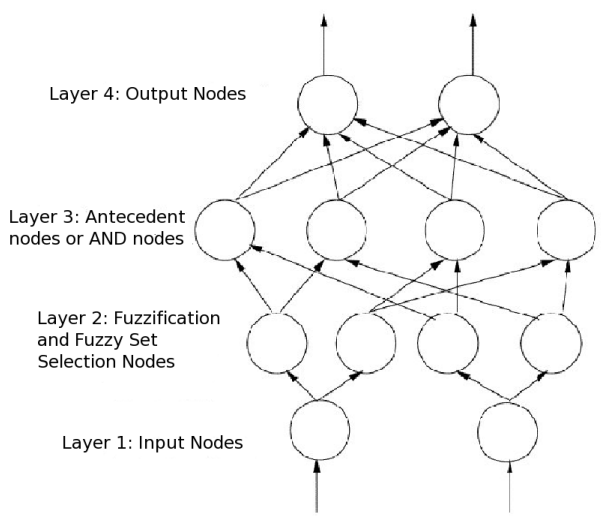

Fig. 1. The network structure for classification

number of nodes in layer 2 will be $N^{2}=\sum_{i=1}^{s} N_{i}$. This layer is also responsible for analysis of input domain for discontinuity. In our simulation we have used the bell-shaped Gaussian function. All connections between nodes in layer 1 and layer 2 is unity and the output of a node in layer 2 is

$$
\bar{z}_{n}=\exp \left\{-\frac{\left(z_{p}-\mu_{n}\right)^{2}}{\sigma_{n}^{2}}\right\} .
$$

The subscript $n$ denotes the $n$th fuzzy set of the linguistic variable $x_{p} \cdot \mu_{n}$ and $\sigma_{n}$ are the mean and spread of the Gaussian membership function representing a linguistic term of $x_{p}$ associated with the node $n$ of this layer.

For identification of null fuzzy sets we use a different modulator function for each fuzzy set (linguistic term) of each feature. The idea is that, the null linguistic terms should always produce a membership value of 0 . Moreover, this will lead the rules involving null fuzzy sets to be fired with strength 0 . This is realized by modulating the outputs of the nodes in the following way

$$
z_{n}=\bar{z}_{n} \times\left(1-e^{-\nu_{n}^{2}}\right)
$$

The parameter $\nu_{n}$ can be learnt using error back-propagation. We can see that when $\nu_{n}^{2}$ takes a large value then $z_{n}$ tends to $\overline{z_{n}}$. On the other hand, for small values of $\nu_{n}^{2}, z_{n}$ tends to 0 . Therefore, after learning, $\nu_{n}^{2}$ should obtain large values for non-null fuzzy sets whereas, it should take small values for null fuzzy sets.

Layer 3 is responsible for performing intersections of the input fuzzy sets of the fuzzy rules. Product operation cannot be chosen for this purpose, because it will produce a very small number as the firing strength of the rules. This does not allow the output nodes to be fired with the desired strength [4. Hence, we used a soft and differentiable version of min regarded as softmin as in 4]:

$$
\operatorname{softmin}\left(x_{1}, x_{2}, \ldots, x_{s}, q\right)=\left(\frac{x_{1}^{q}+x_{2}^{q}+\ldots+x_{s}^{q}}{s}\right)^{\frac{1}{q}} .
$$


As $q \rightarrow-\infty$, softmin tends to the minimum of all $x_{i} \mathrm{~s}, i=1,2, \ldots, s$. For all the simulations we have used $q=-12$. The output of the $m$ th node of this layer is

$$
\overline{z_{m}}=\left(\frac{\sum_{n \epsilon P_{m}} z_{n}^{q}}{\left|P_{m}\right|}\right)^{\frac{1}{q}}
$$

where $P_{m}$ is the set of indices of the nodes in layer 2 connected to node $m$ of layer 3 .

This layer also learns the utility factors corresponding to a fuzzy rule. The utility factor of the $m$ th node of this rule is denoted by $U_{m}$. To keep the utility factors non-negative, we calculate them as $U_{m}=u_{m}^{2}$ as negative utility factors are difficult to interpret. We modulate the output of the nodes in this layer in such a manner that the less significant rules should always produce an output close to zero. This is realized by calculating the output of the $m$ th node of this layer as

$$
z_{m}=z_{m}^{-} u_{m}^{2}
$$

The utility factor $u_{m}$ is initialized with a small value (say, 0.0001) for each node in this layer.

Layer 4 is the output layer of the network. Each node of this layer represents the consequent of the fuzzy rules. Each node of this layer picks up only one antecedent based on the maximum agreement with facts in terms of product of firing strength and certainty factor. This represents a fuzzy union or OR operation implemented by the max operator. The output of node $l$ of layer 4 is given by

$$
z_{l}=\max _{m \epsilon P_{l}}\left(z_{m} g_{l m}^{2}\right)
$$

where $P_{l}$ represents the set of indices of nodes in layer 3 connected to node $l$ in layer 4 .

\section{$3 \quad$ Learning Rules and Fuzzy Set Modulators}

The training phases are designed to minimize the error function

$$
e=\frac{1}{2} \sum_{i=1}^{N} E_{i}=\frac{1}{2} \sum_{i=1}^{N} \sum_{l=1}^{c}\left(y_{i l}-z_{i l}\right)^{2}
$$

where $c$ is the number of nodes in layer 4 and $y_{i l}$ and $z_{i l}$ are the target and actual outputs of node $l$ in layer 4 for input sample $x_{i} ; i=1,2, \ldots, N$. We can drop the subscript $i$ without loss.

The delta for layer 4 is given by

$$
\delta_{l}=-\left(y_{l}-z_{l}\right)
$$

Then delta for layer 3 becomes

$$
\begin{aligned}
\delta_{m} & =\sum_{l \epsilon Q_{m}} \delta_{l} g_{l m}^{2}, & & \text { if } z_{m} g_{l m}^{2}=\max _{m^{\prime}}\left\{z_{m^{\prime}} g_{l m^{\prime}}^{2}\right\} \\
& =0, & & \text { otherwise }
\end{aligned}
$$


where, $Q_{m}$ is the set of indices of the nodes in layer 4 connected to node $m$ of layer 3 .

Similarly, the delta for layer 2 is calculated as

$$
\delta_{n}=\sum_{m \in R_{n}} \delta_{m} u_{m}^{2}\left(\frac{z_{m} z_{n}^{q-1}}{\sum_{n \in P_{m}} z_{n}^{q}}\right) .
$$

where, $R_{n}$ is the set of indices of nodes in layer 3 connected to node $n$ in layer 2 .

Now, having calculated the $\delta$ for each layer, we can calculate the equations for updating weights $g_{l m}$, utility factors $u_{m}$ and modulators for input fuzzy sets $\nu_{n}$. For updating weights we calculate,

$$
\begin{aligned}
\frac{\partial E}{\partial g_{l m}} & =\sum_{l \epsilon Q_{m}} 2 \delta_{l} z_{m} g_{l m}, & & \text { if } z_{m} g_{l m}^{2}=\max _{m^{\prime}}\left\{z_{m^{\prime}} g_{l m^{\prime}}^{2}\right\} \\
& =0, & & \text { otherwise }
\end{aligned}
$$

Where, $Q_{m}$ is the set of indices of nodes in layer 4 connected to node $m$ in layer 3 .

For updation of utility factors,

$$
\frac{\partial E}{\partial u_{m}}=2 \partial_{m} u_{m}\left(\frac{\sum_{n \epsilon P_{m}} z_{n}^{q}}{\left|P_{m}\right|}\right)^{\frac{1}{q}}
$$

Where, $P_{m}$ is the set of indices of nodes in layer 2 connected to node $m$ in layer 3 .

Similarly, for updating $\nu_{n}$ we calculate,

$$
\left.\frac{\partial E}{\partial \nu_{n}}=2 \delta_{n} \nu_{n} \exp \left\{-\left(\left(\frac{z_{p}-\mu_{n}}{\sigma_{n}}\right)^{2}+\nu_{n}^{2}\right)\right)\right\}
$$

where, the node $n$ in layer 2 is connected to node $p$ in layer 1 .

The weights are updated during learning. Initially the weights are assigned some random values in the range $[0,1]$. As weights are updated the weights connecting the actecedtents to its proper consequent get larger values and the rest smaller values resulting in minimization of total system error.

Initially all the $\nu_{n}$ and $u_{m}$ are assigned a very small positive value (say 0.0001 ) so that all the nodes in layer 2 and layer 3 produce an output close to 0 . Thus, all the fuzzy sets are initially considered to be null sets and all rules as less significant ones. As learning proceeds, the fuzzy sets necessary to reduce the system error, i.e, the non-null fuzzy sets, have their $\nu_{n}$ s raised to larger values and the rules necessary to reduce the system error have their $u_{m}$ increased to higher values. Hence, only the non-null fuzzy sets and significant fuzzy rules are allowed to be passed throgh the network.

\section{Results}

The network for pattern classification was tested on many artificially generated data sets. Here we have shown only the results of XOR data. There are two 


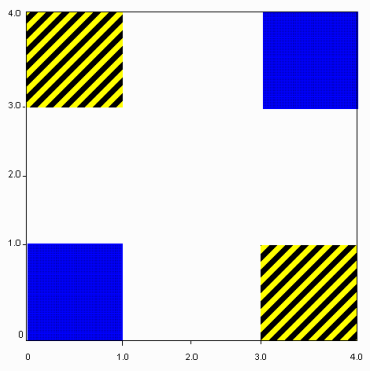

Fig. 2. Plot of XOR data

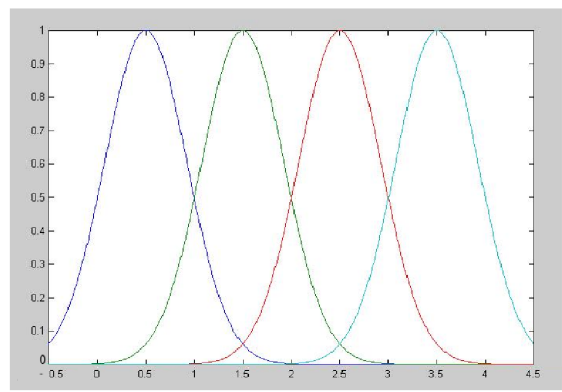

Fig. 3. Plot of fuzzy sets of input variables $x_{1}$ and $x_{2}$ for XOR data

Table 1. Initial architeture of the neuro-fuzzy system for XOR

\begin{tabular}{|c|c|}
\hline Layer no & no of nodes \\
\hline 1 & 2 \\
2 & 8 \\
3 & 16 \\
4 & 2 \\
\hline
\end{tabular}

input features $x_{1}, x_{2}$ and two different classes $c_{1}, c_{2}$. The plot of the XOR data is shown in figure 2. We take four fuzzy sets for both $x_{1}$ and $x_{2}$ as shown in figure 3. The initial network architecture is shown in table 1.

In phase 1 the network was trained for 300 epochs with $\eta=0.1, \theta=0.01$ and $\chi=0.01$. The values for modulators for the input fuzzy sets thus obtained are shown in table 2. Accordingly fuzzy sets $x_{12}, x_{13}, x_{22}$ and $x_{23}$ are removed from the system. In Phase 2 learning is performed with $\eta=0.1$ and $\theta=0.01$ for 300 epochs. The utility factors obtained after this phase are shown in table 3. Clearly, all the four rules are significant and are to be kept.

Hence, from an intial value $4 \times 4 \times 2=32$ the number of rules was ultimately reduced to $2 \times 2 \times 1=4$. Thus an $87.5 \%$ reduction in the number of rules was 
Table 2. Values of $\nu_{n}$ for different linguistic terms of the input features for XOR

\begin{tabular}{|c|c|c|c|c|c|c|c|c|}
\hline & $x_{11}$ & $x_{12}$ & $x_{13}$ & $x_{14}$ & $x_{21}$ & $x_{22}$ & $x_{23}$ & $x_{24}$ \\
\hline \hline$\nu_{n}$ & 1.39 & 0.18 & 0.24 & 1.98 & 1.16 & 0.23 & 0.20 & 1.22 \\
$1-e^{-\nu_{n}^{2}}$ & 0.86 & 0.03 & 0.06 & 0.98 & 0.74 & 0.06 & 0.04 & 0.77 \\
\hline
\end{tabular}

Table 3. Values of utility factors for the fuzzy rules for XOR

\begin{tabular}{|l|l|l|}
\hline & $x_{11}$ & $x_{14}$ \\
\hline \hline$x_{21}$ & 1.91 & 2.14 \\
\hline$x_{24}$ & 1.94 & 1.97 \\
\hline
\end{tabular}

achieved. Moreover, the system could recognize the points not lying to any class even though it was not trained for that. The system was tested with randomly chosen 1000 input points and there were 6 missclassifications with the threshold for belonging to a class being 0.8 resulting the percentage of error $0.6 \%$. There were no missclassifications when the threshold was reduced to 0.6.

\section{Conclusion}

We demonstrated the methodologies to remove the null fuzzy sets and less significant rules from the system after identifying them apart from revisiting the methodologies for pruning the incompatible rules. The system (for pattern classification) is also capable of saying "Don't know" as output when the corresponding sample does not lie in any of the classes the system was trained for [5]. We did not tune the parameters of the fuzzy membership functions which can improve the system performance further. No guideline is also provided to analyze the domains of the output features. We are unable to find a single modulator function which can do both fuzzy set selection as well as feature selection by eliminating all the fuzzy sets corresponding to a redundant feature.

\section{References}

1. Pal, N.R., Pal, T.: On rule pruning using fuzzy neural networks. Fuzzy Sets Syst. 106, 335-347 (1999)

2. Shann, J.J., Fu, H.C.: A fuzzy neural network for rule acquiring on fuzzy control systems. Fuzzy Sets Syst. 71, 345-357 (1995)

3. Chakraborty, D., Pal, N.R.: Integrated Feature Analysis and Fuzzy Rule-Based System Identification in a Neuro-Fuzzy Paradigm. IEEE Trans. Systems Man Cybernetics 31, 391-400 (2001)

4. Chakraborty, D., Pal, N.R.: A Neuro-Fuzzy Scheme for Simultaneous Feature Selection and Fuzzy Rule-Based Classification. IEEE Trans. on Neural Networks 15(1), 110-123 (2004) 
5. Chakraborty, D., Pal, N.R.: Making a Multilayer Perceptron Network Say- "Don't Know" When It Should. In: ICONIP, Orchid Country Club, Singapore (November 18-22, 2002)

6. Ishibuchi, H., Nakashima, T., Morisawa, T.: Voting in fuzzy rule-based systems for pattern classification problem. Fuzzy Sets Syst. 103, 223-238 (1999)

7. Krishnapuram, R., Lee, J.: Propagation of uncertainty in neural networks. In: Proc. SPIE Conf. Robot, Computer Vision, Bellingham, WA, pp.377-383 (1988)

8. Nozaki, K., Isibuchi, H., Tanaka, H.: Adaptive fuzzy rule-based classification systems. IEEE Trans. Fuzzy Syst. 4, 238-250 (1996) 